Ensayos/Essays

\title{
Planeación Local para enfrentar el Desafío de las Enfermedades Crónicas en Pasto, Colombia
}

\section{Local planning to tackle the threat of the chronic diseases in Pasto, Colombia}

Diego I. Lucumí1,2, Alejandra Gutiérrez¹ , José Moreno , Luis F. Gómez¹, Nancy Lagos ${ }^{3}$, Martha Rosero ${ }^{3}$ y Carmen H. Betancourt ${ }^{3}$

1 División Salud Fundación FES Social. Bogotá, Colombia. dilucumi@fundacionfes.org, amgutierrezc@fundacionfes.org, jjmorenom@fundacionfes.org, lfgomez@fundacionfes.org

2 Escuela de Medicina. Universidad Pedagógica y Tecnológica de Colombia.

3 Dirección Municipal de Salud de Pasto, Colombia. nancylagos70@gmail.com, roseromar@latinmail.com, carmebesa@yahoo.com

Recibido 12 Junio 2007/Enviado para modificación 23 Diciembre 2007/Aceptado 28 Marzo 2008

\section{RESUMEN}

Las enfermedades crónicas son un creciente problema de salud pública en el mundo. En países como Colombia, estas enfermedades son una causa importante de morbimortalidad y su tendencia es ascendente. Ante esta situación, diversas entidades han recomendado planear y conducir acciones para su prevención; sin embargo, existen pocas iniciativas con este propósito, lo cual refleja una situación que ha sido denominada como "la epidemia negada de las enfermedades crónicas".

Este artículo describe la experiencia de formulación y los contenidos de un plan para la prevención de estas enfermedades en la ciudad colombiana de Pasto, en cuyo proceso se utilizó un modelo de planificación propuesto por la Organización Mundial de la Salud. El plan busca disminuir en los próximos diez años la prevalencia de los tres principales factores de riesgo para enfermedades crónicas entre 5 y 10 puntos porcentuales, para lo cual se proponen acciones de política pública, en ámbitos de la vida cotidiana, servicios de salud y la generación de evidencia para la toma de decisiones a través de un observatorio de enfermedades crónicas. Esta experiencia destacó la importancia y factibilidad de estos procesos en el contexto local e identificó la articulación con el nivel nacional como uno de los principales desafíos que deberán ser tenidos en cuenta para el desarrollo de este tipo iniciativas.

Palabras Clave: Enfermedad crónica, planificación en salud, promoción de la salud (fuente: DeCS, BIREME).

\section{ABSTRACT}

Chronic diseases are increasing problem of public health in the world. In countries such as Colombia these diseases are an important cause of morbidity and mortality. 
Taking into account this situation several organizations have recommended planning and conducting actions to prevent chronic diseases, however there are scarce initiatives addressed to this purpose which reflect a reality that has been named as the "neglected epidemic of chronic diseases". This article describes the experience of formulation of a local plan for the prevention of chronic diseases in the Colombian city of Pasto where was used a planning model developed by the World Health Organization. The goal of this plan is reduce in the following ten year the prevalence of the three main risk factors for chronic disease between 5 to $10 \%$ addressing the actions in public policies, contexts for the daily life, health services and the generation of evidence to make decisions through an observatory in chronic diseases. This experience showed up the importance and feasibility of this process at the local context and identified the coordination with the national level as one of the main challenges to be considered to carry out these initiatives.

Key Words: Chronic disease, health planning, health promotion (source: $\mathrm{MeSH}$, NLM).

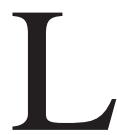

as enfermedades crónicas son la primera causa de muerte a nivel mundial y generan una importante carga social, económica y de discapacidad $(1,2)$. Así lo demuestra el hecho que de 58 millones de muertes ocurridas en el mundo en 2005, 15 millones fueron por estas enfermedades en menores de 70 años (2).

En países como Colombia, las enfermedades crónicas son una causa importante de morbimortalidad y su tendencia es al aumento (1-5). Estos países se caracterizan por la convivencia simultánea de estas enfermedades con las de tipo infeccioso, rápidos procesos de urbanización y transición demográfica y por contar con sistemas de salud poco preparados para enfrentar el desafío de las condiciones crónicas $(1,3)$.

Ante esta situación, diversos organismos han realizado llamados para la acción. Sin embargo, persiste un bajo reconocimiento por parte de tomadores decisiones y de la sociedad de la necesidad de planear acciones para la prevención de las enfermedades crónicas $(1,6)$, lo que puede estar vinculado a creencias erróneas tales como el que estas enfermedades no son una causa relevante de muerte en países pobres, su mortalidad se concentra en adultos mayores y en población con altos ingresos, se carece de acciones costo-efectivas para su prevención y las existentes son difíciles de implementar $(1,3,6)$. De otro lado, no se reconoce como prioridad el desarrollo de acciones con los niños y adolescentes, obviando el conocimiento acumulado sobre la historia natural de estas enfermedades (7). 
En Colombia, esta situación se refleja en la ausencia de políticas públicas nacionales para la prevención de las enfermedades crónicas, al igual que en la carencia de un sistema de vigilancia que provea información útil y oportuna para la toma de decisiones; panorama que encaja con lo que ha se ha denominado la "epidemia negada de las enfermedades crónicas" (8).

Ante la limitada difusión de iniciativas dirigidas a planear las acciones para reducir o controlar las enfermedades crónicas, este artículo describe una iniciativa llevada a cabo en un municipio colombiano, refiriendo el contexto local en el que se formuló, sus fortalezas, limitaciones y desafíos.

\section{Contexto local}

El municipio de Pasto, capital del departamento de Nariño, está ubicado al sur occidente de Colombia. Tiene 431144 habitantes, 89,7 \% habita en la zona urbana y el resto en la rural. Cerca de $30 \%$ de la población del municipio está entre los 5 y 19 años de edad y 26,6 \% de los habitantes no tiene sus necesidades básicas satisfechas (9).

Situación de las enfermedades crónicas. En el año de 2004, la Dirección Municipal de Salud de Pasto (DMS), estimó la prevalencia de los factores de riesgo para enfermedades crónicas en la población adulta del área urbana (10). El estudio reveló que $21 \%$ de los habitantes fumaban cigarrillo, $30 \%$ auto-reportó sobrepeso, $23 \%$, caminaban menos de 10 minutos diarios tres o más días a la semana, 31,5 \% consumían fruta entera y 14,8 \% consumían vegetales diariamente. En ese mismo año las enfermedades cardiovasculares y la diabetes fueron responsables del $32 \%$ de la morbilidad en la ciudad, mientras la hipertensión arterial y el infarto agudo de miocardio fueron las principales causas de muerte, acumulando $18.3 \%$ del total (11).

Antecedentes en la prevención de enfermedades crónicas en Pasto. Como en la gran mayoría de ciudades colombianas, en Pasto, la prevención de las enfermedades crónicas no había sido una prioridad en la planeación local hasta cuando se incluyó en el Plan de Desarrollo Municipal "Pasto Mejor" 2004-2007 un objetivo dirigido a la reducción de estas enfermedades, a partir de lo cual en el año 2005 la DMS tomó la decisión de formular el Plan Local para la Prevención de las enfermedades crónicas (PLIPEC).

Modelo de planificación

Para orientar el proceso de planificación se usó el Marco Progresivo para la Prevención de Enfermedades Crónicas (MPPEC) propuesto por la Organi- 
zación Mundial de la Salud-OMS (Figura 1), que contempla seis principios: complementariedad y subsidiariedad entre niveles, intersectorialidad, abordaje multifactorial de riesgos, desarrollo de acciones poblacionales e individuales, factibilidad y orientación hacia la reducción de la inequidad (12).

Si bien este referente coincide con otros modelos de planeación usados en salud pública (13), tiene la ventaja de centrarse en la planeación de acciones para prevenir y controlar las enfermedades crónicas. De otro lado, al no definir aspectos específicos para la implementación de cada una de las fases descritas, puede adecuarse a diferentes contextos (12), lo cual es considerado como un atributo importante de estos modelos (14).

Figura 1. Marco Progresivo para la Prevención de Enfermedades Crónicas

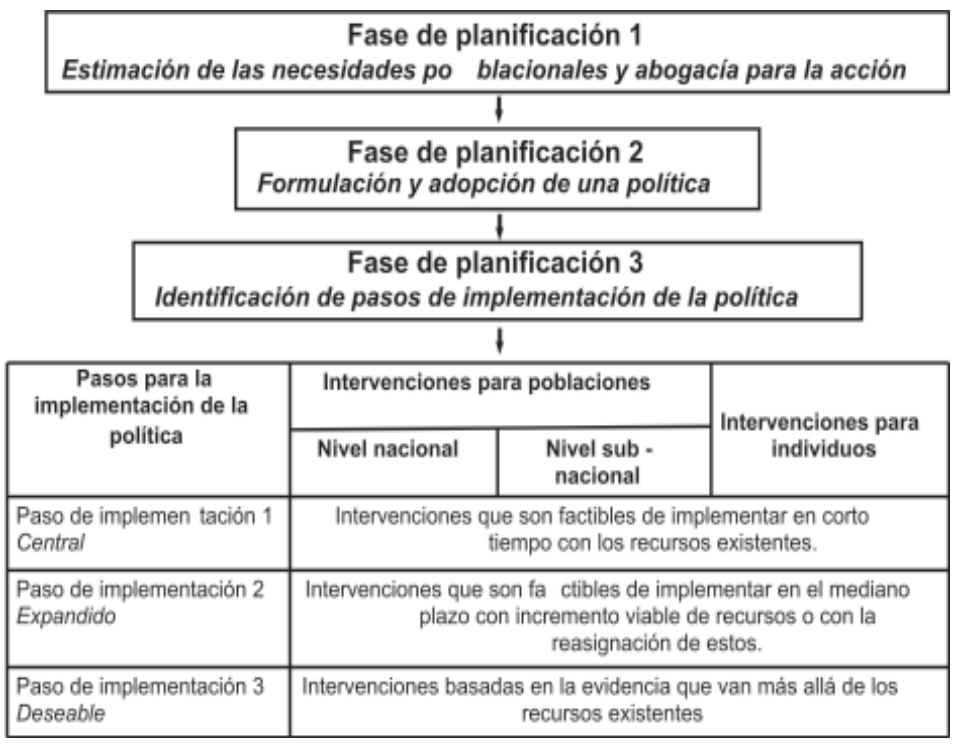

Traducido y publicado con autorización de la OMS. Fuente: World Health Organization. Preventing chronic disease: a vital investment: WHO global report. Geneva: World Health Organization; 2005.

Descripción del proceso de formulación

El proceso de formulación se desarrolló entre enero y agosto de 2006 bajo la orientación de un grupo de investigación con experiencia en enfermedades crónicas, en conjunto con funcionarios de la DMS. Para establecer el alcance de las acciones desarrolladas previamente, se revisaron documentos técnicos que dieran cuenta de las acciones ejecutadas y se condujeron entrevistas semiestructuradas con actores del gobierno y la comunidad. 
Estas actividades llevaron a concluir que era posible avanzar a la segunda fase del MPPEC, considerando que se contaba con información suficiente sobre las condiciones crónicas en Pasto y ya se había avanzado en las actividades de abogacía, por lo cual se definieron las actividades que se describen a continuación.

Elaboración y socialización del marco conceptual. Se actualizó la evidencia sobre el desarrollo de acciones poblacionales para la prevención de enfermedades crónicas, a partir de la revisión de artículos científicos y documentos de la OMS y sus representaciones en América y Europa.

Con el liderazgo y participación de las autoridades municipales tuvo lugar un taller de socialización para posicionar el proceso de formulación del plan, discutir su marco conceptual preliminar y lograr el compromiso de diferentes sectores de participar en su formulación y posterior implementación.

Reconocimiento de acciones, recursos y fuentes de información. Se realizó un reconocimiento de los recursos locales como un paso previo para establecer los objetivos y contenidos del plan, para lo cual se definieron y reconocieron las acciones y recursos, así como las fuentes de información. En cuanto a lo primero, se partió por identificar las acciones del Plan de Desarrollo Municipal 2004-2007 consideradas como relevantes para la prevención de las enfermedades crónicas y aquellas desarrolladas por actores no gubernamentales. En ambos casos, a través de entrevistas semi-estructuradas, se constató la operación de los programas o actividades y se corroboró la disponibilidad de sus responsables de participar en el proceso.

El reconocimiento de fuentes de información identificó fuentes públicas y privadas que pudieran aportar de manera rutinaria o esporádica datos sobre los determinantes, factores de riesgo o morbimortalidad relacionada con las enfermedades crónicas en la ciudad.

Concertación intersectorial. A partir de los acuerdos alcanzados en el taller de socialización, el equipo coordinador del proceso de formulación del plan conjuntamente con los directores municipales de salud y planeación definieron la vigencia, objetivos, metas, estrategias y mecanismos de gestión del plan. Estos contenidos, al igual que los demás componentes del plan, fueron discutidos y concertados en un taller en el que participaron 21 instituciones y organizaciones gubernamentales, sociales y académicas. A partir de lo anterior, el documento se ajustó y luego se aprobó y difundió. 
Contenidos del plan

El PLIPEC está compuesto por un marco conceptual y contextual, los antecedentes y el marco estratégico (15). Este último está integrado por el modelo de planeación de las acciones, la meta general, la población objetivo, los componentes y los mecanismos de gestión.

Para orientar las acciones del PLIPEC se seleccionó el Enfoque Multinivel para la Salud Comunitaria (Multilevel Approach to Community Health-MAT$\mathrm{CH}$ ) (13), el cual es útil para orientar programas de promoción de la salud cuando se parte de un objetivo predeterminado (prevención de las enfermedades crónicas.

La meta establecida contempla que para el año 2015 la ciudad habrá incrementado la prevalencia de actividad física y de alimentación saludable entre 7 a 10 puntos porcentuales y habrá reducido la prevalencia de consumo de cigarrillo entre 5 a 7 puntos porcentuales en la población de 15 a 29 años de edad. Para ello, se definió como población objetivo el grupo de 5 a 19 años de edad, en quienes se hará la evaluación al finalizar los 10 años de vigencia del plan.

Para desarrollar las acciones que permitan alcanzar la meta propuesta se definieron cuatro componentes interdependientes. El componente de política y ambiente busca la implementación y evaluación de políticas y la generación de ambientes que soporten modos y condiciones de vida que protejan contra la aparición de enfermedades crónicas. El componente de acciones colectivas se dirige a la planeación, desarrollo, implementación y rediseño de programas poblacionales que aborden los determinantes de las enfermedades crónicas y promuevan sus factores protectores en ámbitos cotidianos (hogar, comunidad, trabajo, escuela).

El componente de atención sanitaria se centra en el conjunto de organizaciones, procesos y recursos para desarrollar acciones en los tres niveles de prevención desde una perspectiva de atención primaria. Por último, el observatorio de enfermedades crónicas tiene como objetivos identificar, recoger, procesar, analizar y difundir información relacionada con estas enfermedades, a partir de la investigación, la vigilancia en salud pública y el monitoreo.

Finalmente, para la gestión del PLIPEC se definieron tres niveles. El primero es el responsable directo de ejecutar las actividades que se definan en cada componente. El segundo tiene funciones de coordinación y seguimiento, mientras el tercero realiza la orientación política del plan. 


\section{DISCUSIÓN}

La formulación del PLIPEC es coherente con el reconocimiento existente, acerca de la necesidad de generar una respuesta política intersectorial de largo plazo basada en la evidencia para controlar y prevenir las enfermedades crónicas $(1,6,12,16,17)$. De otro lado, este documento constituye un instrumento de acción y control del Estado en el nivel local, en la medida que define las acciones generales que deben ser desarrolladas para alcanzar las metas previstas y propone la difusión de información como uno de los mecanismos que contribuyen a promover el control social.

Como se ha descrito, la formulación de estos planes en países como Colombia plantea varios desafíos $(12,18)$. Por un lado, la prevención de las enfermedades crónicas debe competir con múltiples intereses y prioridades en salud pública. De igual forma, se requiere que las enfermedades crónicas sean posicionadas en la agenda de diversos actores y se apropien modelos de acción científicamente válidos, culturalmente apropiados y sensibles a los recursos locales (18).

Una de las principales fortalezas del PLIPEC es ser una de las primeras experiencias reportadas en América Latina que ha usado el MPPEC como modelo de planificación. En este sentido se debe destacar que el uso de bases teóricas para planear, implementar y evaluar acciones de promoción de la salud contribuye a orientar los procesos, promueve el uso efectivo y eficiente de recursos, genera credibilidad e incrementa el éxito de las acciones $(14,19)$.

Adicionalmente, se adoptó un enfoque integrado caracterizado por reconocer la conglomeración de factores de riesgo, por no se centrarse en la enfermedad, basarse en una perspectiva ecológica, una acción intersectorial en varios niveles y un proceso colaborativo (20). De otro lado, la interacción de funcionarios públicos con investigadores permitió contar con diferentes perspectivas, llevó a proponer acciones más coherentes con las dinámicas locales y a establecer un vínculo real entre la evidencia acumulada a través de la investigación y la toma de decisiones.

Estas fortalezas se expresan en que aunque no se ha evaluado el PLIPEC, se pudo verificar que cumple de forma general con los nueve criterios del instrumento para la evaluación de la calidad de planes para la prevención de enfermedades crónicas propuesto por Butterfoss $(15,21)$. 
Las principales limitaciones de esta experiencia están relacionadas con la falta de estudios que caractericen en forma completa el problema de las enfermedades crónicas en Pasto, al igual que la carencia de evaluaciones sobre los resultados de las acciones desarrolladas antes del plan. Para contrarrestar estas limitaciones, el funcionamiento del observatorio se constituye en una prioridad en la implementación del PLIPEC.

Frente a los desafíos futuros, se hace necesario establecer mecanismos que permitan la continuidad de la participación intersectorial, garanticen la disponibilidad de los recursos requeridos para la implementación, favorezcan la construcción de capacidad local y faciliten la articulación de esta iniciativa con el nivel nacional $(6,12)$.

Aspectos críticos para la implementación del PLIPEC son la falta de una política pública nacional para la prevención de las enfermedades, la exclusividad que tiene el nivel nacional para establecer ciertas regulaciones y la posibilidad de que se aprueben normas que entren en contraposición con planes locales o afecten negativamente sus objetivos, lo que demuestra el papel destacado que tienen las acciones de abogacía ante diferentes niveles y sectores, para obtener el soporte político, técnico y financiero requerido.

En conclusión, el desarrollo de esta iniciativa en otras ciudades podría ser posible en si se cumplan varios aspectos identificados en esta experiencia. Entre estos se pueden considerar el que exista un reconocimiento político y social de las enfermedades crónicas, el uso de un modelo de planificación, el fuerte liderazgo del sector salud para convocar a sectores no involucrados tradicionalmente en iniciativas que afecten la salud de las población, el contar con funcionarios públicos y actores sociales motivados y con equipos que puedan hacer aportes desde diferentes perspectivas •

Agradecimientos. A la Dirección Municipal de Salud de Pasto por financiar el proyecto que dio origen a este artículo (Contrato 104/2005). A las organizaciones gubernamentales, sociales y académicas que participaron en esta iniciativa.

\section{REFERENCIAS}

1. Yach D, Hawkes C, Gould CL, Hofman KJ. The global burden of chronic diseases: overcoming impediments to prevention and control. JAMA 2004; 291(21):2616-22.

2. Strong K, Mathers C, Leeder S, Beaglehole R. Preventing chronic diseases: how many lives can we save?. Lancet 2005; 366 (9496):1578-82. 
3. Organización Mundial de la Salud. Informe sobre la salud en el mundo 2003. Forjemos el futuro. Ginebra: Organización Mundial de la Salud; 2003.

4. González M, De la Hoz F. Mortalidad por enfermedades crónicas no transmisibles en Colombia, 1990 a 1999. Inf Quinc Epidemiol Nac 2002; 7:205-226.

5. Perel P, Casas JP, Ortiz Z, Miranda JJ. Non-communicable diseases and injuries in Latin America and the Caribbean: Time for action. PLoS Med 2006; 3(9): e344. DOI: 10.1371/ journal.pmed.0030344.

6. World Health Organization. Preventing chronic disease: a vital investment: WHO global report. Geneva: World Health Organization; 2005.

7. Escobar M, Petrásovits A, Peruga A, Silva N, Vives M, Robles S. Mitos sobre la prevención y el control de las enfermedades no transmisibles en América Latina. Salud Pública Mex 2000; 42(1):56-64.

8. Horton R. The neglected epidemic of chronic disease. Lancet 2005; 366 (9496):1514.

9. Necesidades básicas insatisfechas. [Internet] Bogotá D.C.: Departamento Administrativo de Nacional de Estadísticas. Disponible en: http://www.dane.gov.co/ index.php?option=com_content\&task=section\&id=35\&ltemid=72 Consultado 10 de julio de 2006.

10. Dirección Municipal de Salud de Pasto. Factores de Riesgo para Enfermedades Crónicas Municipio de Pasto. San Juan de Pasto: Dirección Municipal de Salud; 2004.

11. Dirección Municipal de Salud de Pasto. Indicadores Básicos 2000-2005. Pasto: Dirección Municipal de Salud; 2005.

12. Epping-Jordan J, Galea G, Tukuitonga C, Beaglehole R. Preventing chronic diseases: taking stepwise action. Lancet 2005; 366(9497):1667-71.

13. McKenzie J, Neiger B, Smeltzer J. Planning, implementing \& evaluating health promotion programs: A primer. Fourth edition. San Francisco (CA): Pearson Benjamin Cummings; 2005.

14. Dunet DO, Butterfoss FD, Hamre R, Kuester S. [Internet]. Using the State Plan Index to evaluate the quality of state plans to prevent obesity and other chronic diseases. Prev Chronic Dis 2005 Apr. Disponible en: http://www.cdc.gov/pcd/issues/2005/apr/ 04_0090.htm Consultado 22 agosto 2006.

15. Dirección Municipal de Salud de Pasto. Plan Integrado Local de Acción para la Prevención de Enfermedades Crónicas en Pasto 2006-2015. Bogota D.C.: Fundación FES Social; 2006.

16. Brownson R, Haire-Joshu D, Luke D. Shaping the context of health: a review of environmental and policy approaches in the prevention of chronic diseases. Annu. Rev. Public Health 2006; 27:341-370.

17. Nishtar S. Prevention of non-communicable diseases in Pakistan: an integrated partnershipbased model. Health Research Policy and Systems 2004; 2:7 doi:10.1186/1478-45052-7.

18. Srinath Reddy K, Shah B, Varghese C, Ramadoss A. Responding to the threat of chronic diseases in India. Lancet 2005; 366(9498):1744-9.

19. Cabrera G. Teorías y modelos en la salud pública del siglo XX. Colombia Médica. 2005;3(35):184-188.

20. Wolbeck Minke S, Smith C, Plotnikoff RC, Khalema E, Raine K [Internet]. The evolution of integrated chronic disease prevention in Alberta, Canada. Prev Chronic Dis 2006 Jul Disponible en: http://www.cdc.gov/pcd/issues/2006/jul/05_0225.htm Consultado 11 de junio de 2007.

21. Butterfoss FD, Dunet DO [Internet]. State Plan Index: a tool for assessing the quality of state public health plans. Prev Chronic Dis 2005 Apr. Disponible en: URL:http://www.cdc.gov/ pcd/ issues/2005/apr/04_0089.htm Consultado 2 de junio de 2007. 